\title{
Reduced Skin Blistering in Experimental Epidermolysis Bullosa Acquisita After Anti-TNF Treatment
}

\author{
Misa Hirose, ${ }^{1}$ Anika Kasprick, ${ }^{1}$ Foteini Beltsiou, ${ }_{1}^{1}$ Katharina Schulze Dieckhoff, ${ }_{1}^{1}$ Franziska Sophie Schulze, ${ }^{1}$ \\ Unni KJSRL Samavedam, ${ }_{1}^{1}$ Jennifer E Hundt, ${ }^{1}$ Hendri H Pas, ${ }^{4}$ Marcel F Jonkman, ${ }^{4}$ Enno Schmidt, ${ }^{1}$ \\ Kathrin Kalies, ${ }^{3}$ Detlef Zillikens, ${ }^{1,2}$ Ralf J Ludwig, ${ }^{1 *}$ and Katja Bieber ${ }^{1 *}$ \\ ${ }^{1}$ Lübeck Institute of Experimental Dermatology, University of Lübeck, Lübeck, Germany; ${ }^{2}$ Department of Dermatology, Allergology \\ and Venereology, University of Lübeck, Lübeck, Germany; ${ }^{3}$ Institute of Anatomy, University of Lübeck, Lübeck, Germany; and \\ ${ }^{4}$ Department of Dermatology, University of Groningen, Groningen, the Netherlands
}

\begin{abstract}
Epidermolysis bullosa acquisita (EBA) is a difficult-to-treat subepidermal autoimmune blistering skin disease (AIBD) with circulating and tissue-bound anti-type VII collagen antibodies. Different reports have indicated increased concentration of tumor necrosis factor $\alpha$ (TNF) in the serum and blister fluid of patients with subepidermal AIBD. Furthermore, successful anti-TNF treatment has been reported for individual patients with AIBD. Here we show that in mice, induction of experimental EBA by repeated injections of rabbit anti-mouse type VII collagen antibodies led to increased expression of TNF in skin, as determined by real-time polymerase chain reaction (PCR) and immunohistochemistry. To investigate whether the increased TNF expression is of functional relevance in experimental EBA, we inhibited TNF function using the soluble TNF receptor fusion protein etanercept (Enbrel) or a monoclonal antibody to murine TNF. Interestingly, mice that received either of these treatments showed significantly milder disease progression than controls. In addition, immunohistochemical staining demonstrated reduced numbers of macrophages in lesional skin in mice treated with TNF inhibitors compared with controls. Furthermore, etanercept treatment significantly reduced disease progression in immunization-induced EBA. In conclusion, increased expression of TNF in experimental EBA is of functional relevance, as both the prophylactic blockade of TNF and the therapeutic use of etanercept impaired induction and progression of experimental EBA. Thus, TNF is likely to serve as a new therapeutic target for EBA and AIBDs with a similar pathogenesis.
\end{abstract}

Online address: http://www.molmed.org

doi: $10.2119 / \mathrm{molmed} .2015 .00206$

\section{INTRODUCTION}

Tumor necrosis factor $\alpha(\mathrm{TNF})$ was originally described as a circulating factor that can cause necrosis of tumors, but has since also been identified as a key regulator of inflammatory responses (1). TNF is typically not detectable in the serum and tissue of healthy individuals; however, elevated serum and tissue levels are found in inflammatory and infectious conditions, and serum levels correlate with the severity of infection $(2,3)$. In accordance with this, TNF blockade has been shown to be an effective therapy in various immune-mediated inflammatory diseases, such as rheumatoid arthritis, psoriatic arthritis, plaque psoriasis, inflammatory bowel disease (i.e., Crohn's disease, ulcerative colitis and intestinal Behçet's disease), ankylosing spondylitis, axial spondyloarthritis, systemic vasculitis and juvenile idiopathic

${ }^{*} R J L$ and KB contributed equally.

Address correspondence to Katja Bieber, Lübeck Institute of Experimental Dermatology, Ratzeburger Allee 160, University of Lübeck, 23582 Lübeck, Germany, Phone: + 49-451-500-50883; Fax: +49-451-500-50888; E-mail: katja.bieber@uksh.de Submitted September 14, 2015; Accepted for Publication December 12, 2016: Published online (www.molmed.org) December 20, 2016.

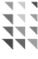

Feinstein Institute
for Medical Research Northwell Health

arthritis (4-8). High levels of TNF also were detected in the wound fluid of chronic nonhealing wounds and induce cytotoxicity (9).

In many chronic inflammatory diseases, TNF inhibition results in the downregulation of abnormal and progressive inflammatory processes, induces rapid and sustained clinical remission, improves quality of life and prevents target organ damage (10). It acts as a potent chemoattractant for neutrophils, induces microvascular leakage and promotes the expression of adhesion molecules on endothelial cells, helping neutrophils to migrate $(11,12)$. Furthermore, TNF is upregulated in activated neutrophils and interacts both synergistically and antagonistically with cytokines of the IL-6 family (13). The subsequent development of biological agents capable of blocking TNF has led to important advances in the pharmacotherapy of such diseases 
and confirmed the concept of a common pathophysiology among immune-mediated inflammatory diseases, with TNF having a predominant role. Five TNF inhibitors have currently been approved for treatment of 1 or more immune-mediated diseases: adalimumab, etanercept (ETA), golimumab, infliximab and certolizumab pegol (6). Because of the role of TNF blockade in many inflammatory diseases, we evaluated its contribution to another immune-mediated inflammatory disease, epidermolysis bullosa acquisita (EBA), a prototypic antibody-dependent and organ-specific autoimmune blistering skin disease (AIBD) (14-17).

The pathogenic relevance of autoantibodies in EBA has been clearly demonstrated $(18,19)$. These autoantibodies are directed against type VII collagen (COL7), a major component of anchoring fibrils. After binding to its target in the skin, a proinflammatory milieu is generated. In different experimental models of EBA, it has been demonstrated that proinflammatory cytokines such as IL-1, granulocyte macrophage colony-stimulating factor (GM-CSF) and antiinflammatory cytokines such as IL-6 are involved in the pathogenesis of EBA $(20,21)$. Together with complement activation (22), this milieu leads to neutrophil extravasation and activation (23) in an Fc $\gamma$ R-dependent manner (24). Reactive oxygen species and proteolytic enzymes are released by neutrophils, resulting in subepidermal blister formation (23). Increased expression of TNF in skin and/or serum of AIBD patients has long been noted (25). Individual case reports show clinical remission of pemphigoid disease after second-line therapy with etanercept $(26,27)$ and effects in patients with pemphigus vulgaris (PV) (28-31). Functional data demonstrated that after transfer of pemphigus patient serum into neonatal mice, TNFR1-/TNFR2-deficient mice developed fewer blisters than wild-type mice (32). In contrast, no data concerning the role of TNF in the pathogenesis of EBA have been published so far. Therefore, we analyzed the effects of TNF blockade on the effector phase of this disease.

\section{MATERIALS AND METHODS}

\section{Experiments with Human Biomaterial}

For determination of TNF expression and cryosections assays, normal human skin as well as lesional and nonlesional skin from EBA and bullous pemphigoid (BP) patients was obtained. Diagnosis was established based on clinical presentation, detection of IgG and/or C3 deposits in direct IF microscopy of perilesional skin biopsies, detection of the corresponding circulating autoantibodies or detection of a u-serrated pattern in direct IF microscopy in the case of EBA patients. All experiments using human samples were approved by the local ethics committee (University of Lübeck, Germany, and University of Groningen, the Netherlands) and were performed according to the Declaration of Helsinki. Blood donors and patients provided written informed consent prior to study participation.

\section{Animal Experiments}

C57BL/6J mice (Charles River) were used for the antibody transfer-induced EBA model with prophylactic application of ETA and monoclonal antibody. B6.SJL-H2s C3c/1CyJ (B6.S) animals were provided by Jackson Laboratories and bred at the University of Lübeck, Germany. Mice were used for experimental EBA models at the age of 8 to $10 \mathrm{wks}$. Animal experiments were approved by local authorities of the Animal Care and Use Committee (Kiel, Germany) and performed by certified personnel.

\section{Induction of Experimental EBA and BP}

Antibody transfer-induced EBA: Rabbits were immunized with a fragment of murine type VII collagen NC1 (mCOL7C). IgG from immune serum was isolated as previously described (33). Mice were injected intraperitoneally (i.p.) $6 \times$ with $5 \mathrm{mg}$ rabbit anti-mCOL7C IgG per mouse for $5 \mathrm{~d}$ consecutively. ETA (Enbrel) was administered i.p. ( $2.5 \mathrm{mg} / \mathrm{kg}$ body weight) $2 \mathrm{~d}$ prior to the initial anti-mCOL7C IgG injection and then every other day ( $7 \times$ total).
Blocking monoclonal antibody to murine TNF (clone MP6-XT22, eBioscience) or isotype antibody (clone eBRG1, eBioscience) with a dose of $150 \mu \mathrm{g} /$ mouse was injected i.p. every other day starting $2 \mathrm{~d}$ prior to the initial rabbit anti-mCOL7C IgG injection and stopping at $\mathrm{d} 8(6 \times$ total $)$.

Immunization-induced EBA: B6.S mice were immunized with $120 \mu \mathrm{g}$ vWFA2 domain of COL7 emulsified (1:2) in TiterMax as described previously, with minor modifications $(34,35)$. Briefly, $60 \mu \mathrm{L}$ of vWFA2/TiterMax emulsion was injected into the foot pad of each hind leg. Starting 3 wks after immunization, mice were randomly allocated into either TNF blocking or solvent treatment groups if they presented erythema, blisters, erosions, crust or alopecia on more than $2 \%$ of their body surface area. Treatment was performed with ETA injected i.p. ( $2.5 \mathrm{mg} / \mathrm{kg}$ body weight) every other day for 6 wks. For clinical score, mice were examined once per week, and body surface areas affected by erythema, blisters, erosions, crust or alopecia were observed. Relative clinical scores were calculated by normalizing the weekly score to the initial clinical score when allocated into treatment groups (weekly clinical score/initial clinical score). Disease severity was defined as the integrated relative clinical score over time (area under the curve).

Local antibody transfer-induced BP: Rabbits were immunized with a fragment of murine type XVII collagen and IgG from immune serum was isolated as previously described (36). Mice were injected once with $1 \mathrm{mg}$ rabbit anti-mCOL17 IgG per mouse into the ear base. ETA (Enbrel) was administered i.p. ( $2.5 \mathrm{mg} / \mathrm{kg}$ body weight) $2 \mathrm{~d}$ prior to the initial anti-mCOL7C IgG injection and then every day ( $4 \times$ total). Ear thickness and scoring were performed at $\mathrm{d} 2$ after IgG injection.

\section{Histopathology and Direct Immunofluorescence Staining}

Biopsies of lesional and perilesional skin were obtained at d 12 of antibody transfer-induced EBA and prepared for 
examination by histopathology and IF microscopy, as described previously (33). In brief, the biopsies collected from mice were fixed in $4 \%$ phosphate-buffered saline (PBS) and buffered formalin, and subsequently sections from paraffinembedded tissues were stained with hematoxylin and eosin. IgG and C3 deposits were detected by direct IF microscopy on frozen sections prepared from tissue biopsies using fluorescein isothiocyanatelabeled antibodies specific to rabbit IgG (Dako) and murine C3 (MP Biomedicals).

\section{Immunohistochemistry and Immunofluorescence}

Immunohistochemical analysis was performed as previously described, with minor modifications (21). Monoclonal antibodies specific for murine TNF (clone MP6-XT22) were purchased from AbD Serotec, anti-human TNF antibody (clone 52B83) was purchased from Abcam and isotype control (mouse reference serum) was purchased from Bethyl Laboratories. Rat monoclonal antibody (clone MOMA-2) for staining of intracellular antigen of mouse macrophages and monocytes was purchased from Acris Antibodies. Serial cryosections $(6 \mu \mathrm{m})$ of organ tissue samples were fixed in absolute acetone for $10 \mathrm{~min}$ at $4^{\circ} \mathrm{C}$ and air-dried before blocking of nonspecific immunoreactive sites with $3 \%$ bovine serum albumin or $5 \%$ normal goat serum (Sigma) in PBS for $20 \mathrm{~min}$ at room temperature. The sections were subsequently incubated with primary antibodies at the appropriate dilution at $4^{\circ} \mathrm{C}$ overnight. Staining was visualized by addition of secondary antibodies (peroxidaseconjugated goat anti-rat IgG, Dianova) and Histogreen Chromogen (Linaris), and the reaction was terminated by washing in tap water. Samples were counterstained with Meyer's hematoxylin and mounted with Vectamount (Vector Laboratories). Alternatively, fluorescent-labeled secondary antibodies $\left(\mathrm{Cy}^{\mathrm{TM}} 3\right.$-conjugated goat anti-mouse IgG [H + L] polyclonal antibody, 1/100 in PBS-T, Jackson Laboratories) were used and the sections were embedded using DAPI and Mowiol 4-88 (Sigma).

\section{Real-time PCR}

Real-time PCR of skin sections was performed as previously described (37). RNA from lesional $(\mathrm{n}=4)$ and nonlesional $(n=3)$ skin from corresponding anatomical sites was obtained using the innuPREP RNA Mini Kit (Analytik Jena). After reverse transcription, cDNA was added to the GPCR MasterMix Plus (Eurogentec) and amplified using an SDS ABI 7000 or SDS ABI 7900 system (Applied Biosystems). TaqMan probes and forward and reverse primers were designed with CloneManager (SciEd); MLN-51 was used as a housekeeping gene. The primers used were $\mathrm{mMLN}-51$ for (5' CCA AGC CAG CCT TCA TTC TTG), mMLN-51rev (5' TAA CGC TTA GCT CGA CCA CTC TG), mTNFfor (5' CCC TCA CAC TCA GAT CAT CTT CTC) and mTNFrev (5' TGG CTC AGC CAC TCC AG).

\section{Bio-Plex}

On d 12 of antibody transfer-induced EBA, serum was obtained from the mice and analyzed for concentrations of cytokines (TNF, IL-6, G-CSF and keratinocyte chemoattractant $[\mathrm{KC}])$. This was performed by a commercial supplier using the Bio-Plex system (Bioglobe $\mathrm{GmbH}$ ).

\section{Reactive Oxygen Species Release Assay}

A Lumitrack high-binding 96-well plate (Thermo Fisher Scientific) was coated with immune complexes (ICs) consisting of human Col7E-F antigen at a final concentration of $2.5 \mu \mathrm{g} / \mathrm{mL}$ and anti-human Col7-IgG1 or anti-human Col7- $\operatorname{Ig} A 2$ antibody at a final concentration of $1.8 \mu \mathrm{g} / \mathrm{mL}$ as described previously (38); $2 \times 10^{5}$ cells were added per well. As controls, antigens or antibodies alone were added to the wells. Just before measurement, luminol (Sigma) was added to the wells and chemiluminescence resulting from reactive oxygen species (ROS) production was measured immediately in a luminescence reader (Wallac 1420 Manager, Victor3). The ROS release was measured for $1 \mathrm{~s}$ per well $66 \times$ for a period of $\sim 3 \mathrm{~h}$ at a constant temperature of $37^{\circ} \mathrm{C}(38)$.

\section{Human Monocytes and Polymorphonuclear Purification}

Human polymorphonuclear (PMN) leukocytes and peripheral blood mononuclear cells (PBMCs) were isolated from whole blood samples using the PolymorphPrep (Progen) gradient according to the manufacturer's instructions. In the next step, human monocytes were purified from the isolated PBMCs by the magnetic cell separation method using a Monocyte Isolation Kit II, human (Miltenyi), according to the manufacturer's instructions. The purity of monocytes and neutrophils was evaluated by fluorescent staining with PE/Cy7 anti-human CD14 antibody (clone HCD14, Biolegend) and fluorescein isothiocyanate anti-human CD16 antibody (BD Biosciences) in a flow cytometer (MACSQuant Analyzer 10, Miltenyi).

\section{Human Cryosection Assay}

Human breast skin biopsies from healthy donors were cut into $6 \mu \mathrm{m}$ sections. The slides were incubated in a humidified air incubator containing $5 \% \mathrm{CO}_{2}$ (Memmert) for $1 \mathrm{~h}$ at $37^{\circ} \mathrm{C}$ with $200 \mu \mathrm{g} / \mathrm{mL}$ anti-human Col7-IgA2 antibody or $1 \times$ PBS for the negative controls. Cryosection assay was performed as previously described (38). In short, a chamber of approximately $0.3 \mathrm{~mm}$ thickness and $0.25 \mathrm{~mL}$ volume was created by covering a slide containing the tissue section with a second slide carrying transparent adhesive tape around its ends and taping them together with Parafilm (Pechiney). Next, $5 \times 10^{6}$ monocytes or PMNs were added to the chamber at a final volume of $150 \mu \mathrm{L}$. After incubation for $2.5 \mathrm{~h}$ at $37^{\circ} \mathrm{C}$, the chamber was disassembled. A washing step with $1 \times$ PBS for $15 \mathrm{~min}$ followed, and the sections were fixed in formalin and stained with hematoxylin and eosin. Images in brightfield mode were captured using a Keyence BZ-9000 fluorescence microscope and BZ-II Viewer software (Keyence). The length of the split formation was assessed by 2 independent and blinded observers. 


\section{Statistical Analysis}

All data are presented as the mean \pm standard error of the mean, unless otherwise indicated. GraphPad Prism 5 and 7 were used to determine significant differences. For comparison of differences between two groups, $t$ test was used, and for analysis of more than two groups, one-way analysis of variance with Bonferroni post-test was applied. A $p$ value $<0.05$ was considered statistically significant.

\section{RESULTS}

TNF Levels Are Increased in the Skin of Experimental and Human AIBD

To investigate a possible effect of TNF during EBA and other AIBDs, we stained three human lesional and nonlesional skin specimens from EBA and BP patients and three normal human skin specimens using anti-TNF antibody. As expected, only inflamed regions (around the blister) showed clear staining for TNF, whereas normal human skin and nonlesional regions were negative for TNF (Figure 1A). To verify these findings in experimental models of EBA, we performed repeated injections of rabbit anti-mCOL7C IgG in mice (39). To verify these findings in mouse models of AIBD, ear samples were collected from mice injected with anti-mCOL7C IgG and with normal rabbit IgG $12 \mathrm{~d}$ after the first injection. By immunohistochemistry staining of the ear skin with rat anti-mouse TNF antibody, we demonstrated increased staining of TNF in skin lesions of diseased mice, while less staining was found in the control skin (Figures 1B, C). Furthermore, TNF mRNA expression in lesional skin of mice with antibody transfer-induced EBA was significantly increased compared to that in healthy skin from the same mice ( $p=0.0382$, Figure 1D). In contrast, the level of TNF in the serum of mice after antibody transfer-induced EBA was similar between mice that received pathogenic IgG and controls ( $p=0.7075$, Figure 1E). These data suggest that tissue-expressed TNF (especially in lesional skin) but not
A

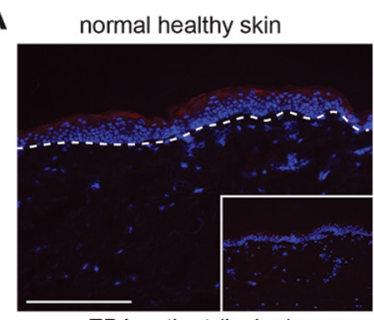

EBA patient (lesion)

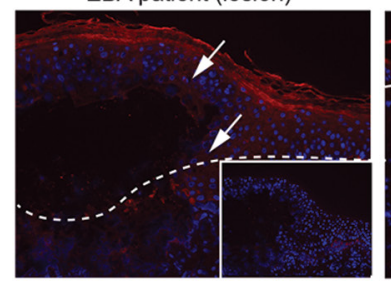

D

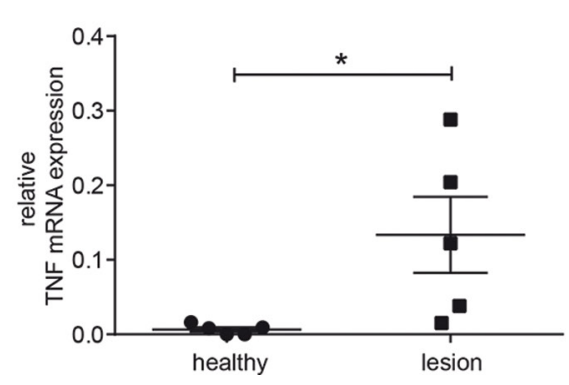

BP patient (lesion)

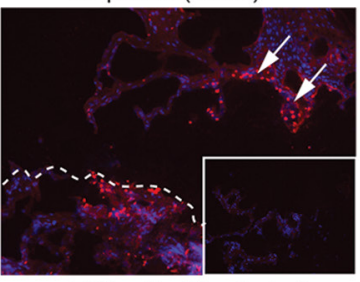

EBA patient (no lesion)

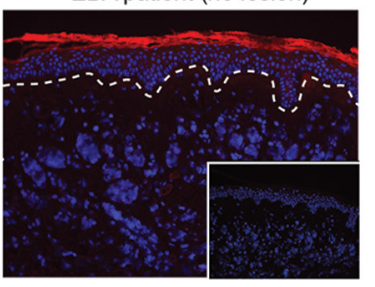

E

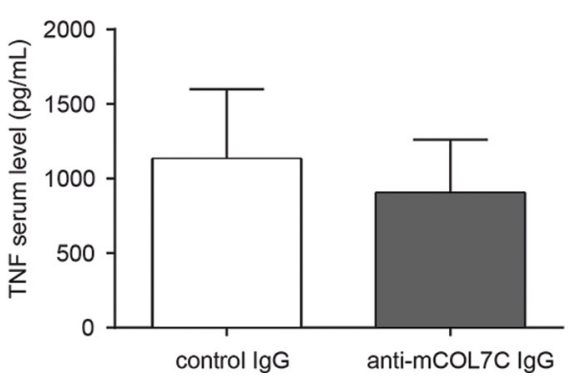

Figure 1. Tissue expression of TNF was increased in experimental EBA. (A) Immunofluorescence staining of skin obtained from human BP and EBA patients and normal human skin. Red color indicates the presence of TNF only in lesional skin from EBA or BP patients. Inlay: isotype control staining. Images shown are representative of three specimens investigated per group. (B,C) Immunohistochemistry staining of ear skin obtained from (B) an anti-mCOL7C IgG-injected mouse and (C) a normal rabbit IgG-injected control mouse (scale bar: $400 \mu \mathrm{M}$ ). Blue color indicates the presence of TNF. (D) Ear samples from lesional skin (lesion, $n=5$ ) and healthy areas (healthy, $n=5$ ) after antibody transfers of anti-mCOL7C lgG were analyzed for mRNA levels of TNF by real-time PCR ( $n=5 /$ group, ${ }^{*} p=0.0382$ ). (E) TNF concentration was measured by Bio-Plex in the serum of antibody transfer-induced EBA (anti-mCOL7C IgG, $n=7$ ) and normal rabbit IgG-injected animals (control IgG, $n=4$ ) $12 \mathrm{~d}$ after the first IgG injection. No significant difference was observed between the two groups.

circulating TNF is increased in experimental EBA and human AIBDs.

\section{Prophylactic TNF Inhibition Reduces Blistering in Antibody Transfer-Induced EBA and BP}

Mice treated with TNF blocking antibody (TNF mAb) and subsequently injected with anti-mCOL7C IgG showed a significant difference regarding disease progression at $\mathrm{d} 8$ and 12 when compared with mice treated with an isotype-control antibody (Figures 2A-D) (d 8, $p=0.029$; d 12, $p=0.009)$. Immunohistochemistry staining revealed reduced monocytes/ macrophages (anti-MOMA-2) and TNF in lesional skin of the TNF mAb-treated group, while blister formation and IgG/C3 deposition at the dermal-epidermal junction were comparable in both groups (Figures 2F-O).

Currently, the contribution of monocytes/macrophages to the development of skin lesions in EBA, in both patients and experimental models, has not been studied in detail. Here, we show that macrophages constitute a substantial percentage 

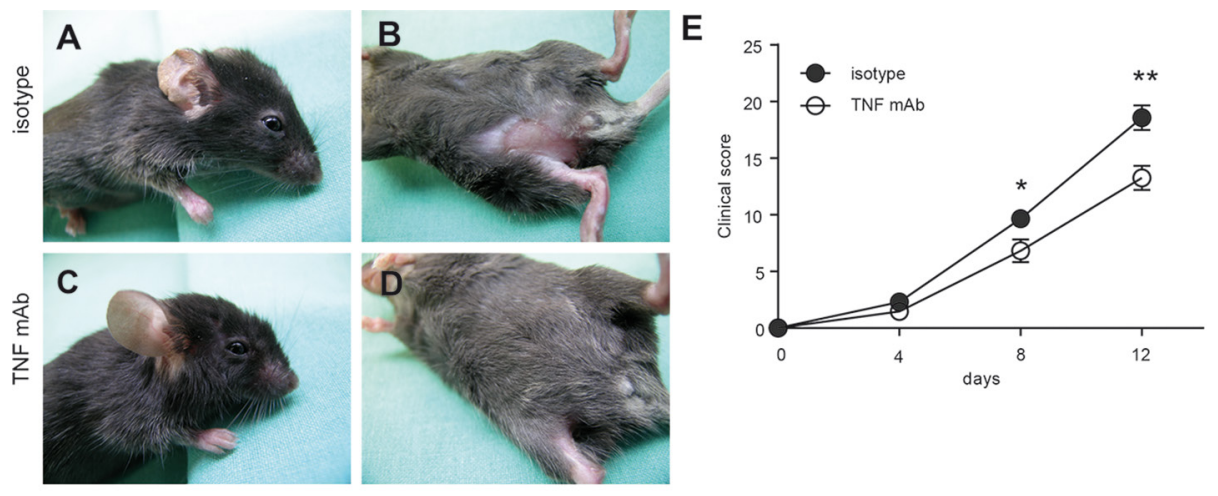

$\lg G$

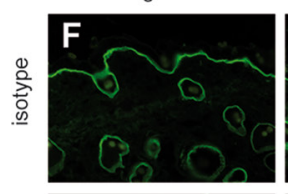

C3

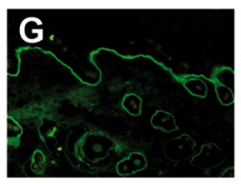

HE

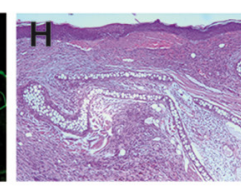

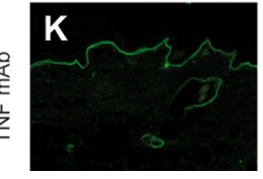

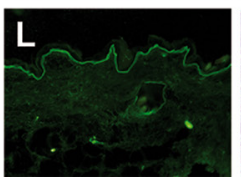

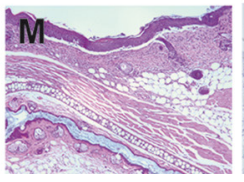

TNF

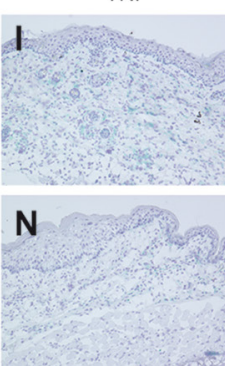

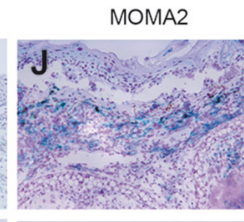

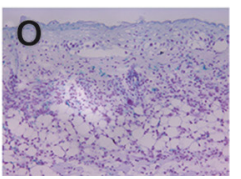

Figure 2. Prophylactic TNF blockade reduced the extent of skin disease in antibody transferinduced EBA. Mice injected with rabbit anti-mCOL7C IgG and treated with $(A, B)$ an isotype antibody $(n=8)$ or ( $C, D)$ a monoclonal antibody to TNF (TNF $m A b, n=6) 2$ d prior to initial anti-mCOL7C IgG injection, followed by five treatments every other day until d 8. Ears and trunks of isotype-treated mice demonstrated erythema and erosions, while less disease was observed in TNF mAb-treated mice. (E) Time course of clinical disease development, shown as the percentage of body surface area affected by blistering $\left({ }^{*} p<0.05\right.$, $\left.{ }^{* *} p<0.01\right)$. Representative ear samples from $(F-J)$ isotype-treated and $(K-O)$ TNF mAbtreated mice with EBA were analyzed histopathologically and immunologically. By direct immunofluorescence microscopy, similar $(F, K) \lg G$ and $(G, L) C 3$ deposits were observed at the dermal-epidermal junction in both experimental groups. Histopathological analysis of lesional skin demonstrated comparable $(\mathrm{H}, \mathrm{M})$ dermal-epidermal separation in both treatment groups, but $(I, N)$ TNF and $(J, O)$ MOMA-2 were detected to a lesser extent in $(\mathrm{N}, \mathrm{O})$ TNF mAb-treated mice compared with $(\mathrm{I}, \mathrm{J})$ isotype-treated mice. Magnification of all sections is $200 x$.

of infiltrated leukocytes in lesional skin obtained from mice with experimental EBA (Figure 2J). To further investigate this point, we compared monocytes/macrophages based on the possibility of their producing reactive ROS after IC stimulation (Supplementary Figure S1A, S1B) and inducing split formation after immune complex formation in an ex vivo assay with human skin (Supplementary Figure S1C). Indeed, besides the known fact that PMNs contribute to this pathogenic effect in AIBDs, monocytes / macrophages are a second cell type that directly contributes to blister formation.
To further investigate the role of TNF in the context of AIBDs, the impact of ETA with known effectiveness against murine TNF was investigated in antibody transfer-induced EBA. For this purpose, C57BL6/J mice were treated with ETA and subsequently anti-mCOL7C IgG. Although all mice developed clinical symptoms of EBA (Figures 3A-D), the disease was significantly milder in ETA-treated mice compared with the control group (d 8, $p=0.03$; d 12, $p=0.005$; Figure $3 E$ ). In addition, circulating cytokine levels were evaluated in the serum obtained from diseased mice treated with either isotype or ETA and TNF $\mathrm{mAb}$. Although serum levels of TNF were unaltered in the TNF intervention groups compared with untreated mice (Figure 1A), levels of IL-6, G-CSF and $\mathrm{KC}$ were reduced after TNF inhibition (Figure 4). To verify the effect of TNF blockage in another mouse model for autoimmune blistering disease, we also injected mice with ETA and subsequently with anti-mCOL17 IgG as an experimental model for BP (40). Again, treatment with ETA showed a significant difference regarding disease progression at $\mathrm{d} 2(p=0.04)$ (Figures $2 \mathrm{~F}-\mathrm{G}$ ).

\section{Therapeutic Treatment with ETA Diminishes the Extent of Blistering in Immunization-Induced EBA}

We next evaluated the therapeutic potential of ETA in mice with already established clinical EBA. B6.S mice were immunized with the vWFA2 domain of COL7C for induction of experimental EBA. Mice that presented at least $2 \%$ of body surface area affected by blistering were randomly allocated to either the ETA or PBS treatment group. Compared to PBS-treated controls, mice that received ETA showed a clinically significantly reduced extent of blistering (Figure 5), indicating the important role of TNF during the pathogenesis of experimental EBA.

\section{DISCUSSION}

In this study, we provide evidence for a proinflammatory role of TNF in experimental models of a prototypic organspecific autoantibody-mediated disease. First, we observed increased TNF expression in the skin of mice that developed experimental EBA and in human BP and EBA patients. Second, pharmacological blockade of TNF by ETA or a murine TNF-blocking $\mathrm{mAb}$, if applied before the onset of skin blisters, led to significantly milder disease progression in anti-mCOL7C IgG-treated mice compared with control mice. Third, in addition to this prophylactic treatment, we further demonstrated that TNF inhibition effectively improved already established 

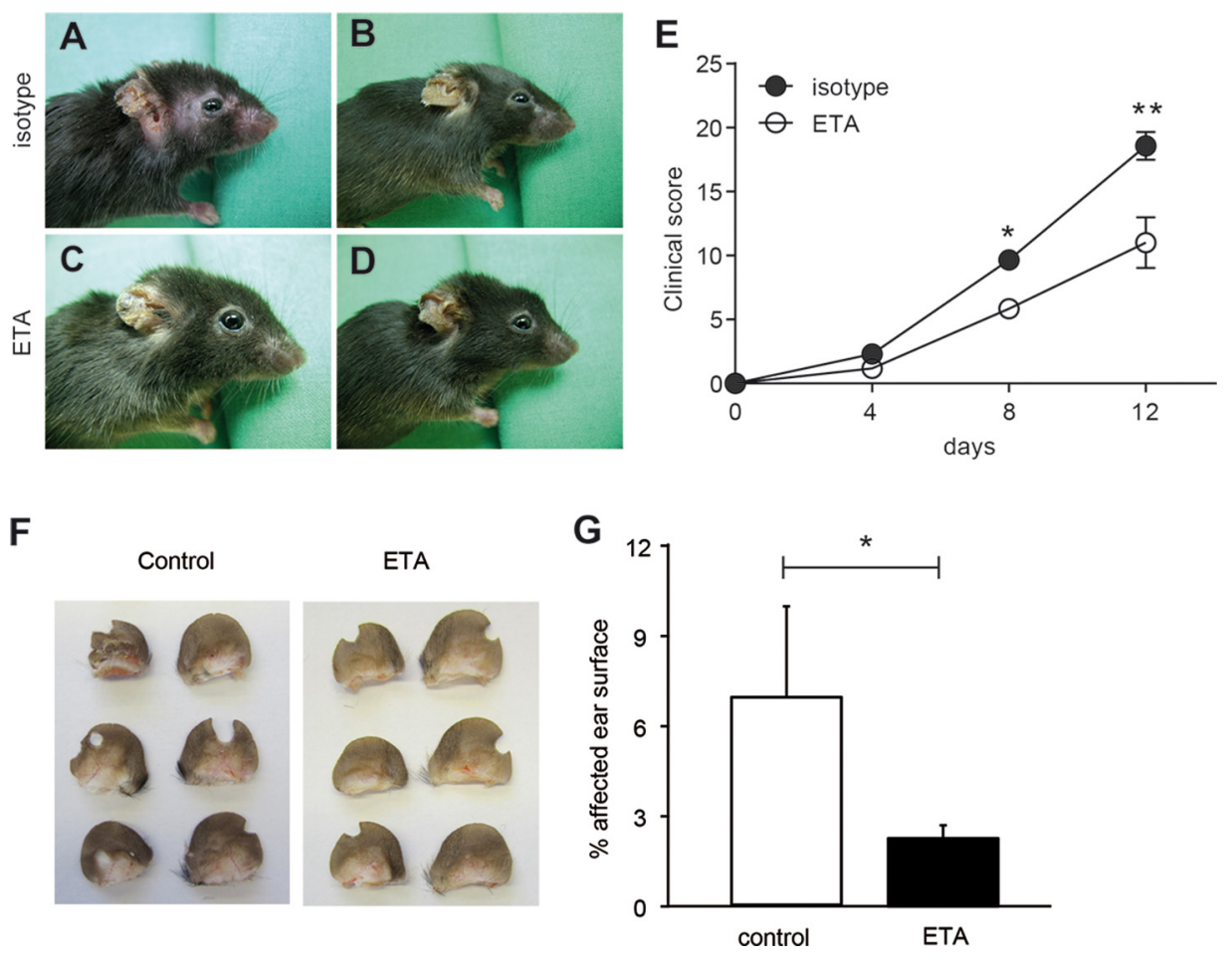

Figure 3. Prophylactic inhibition of TNF using ETA in antibody transfer-induced EBA and BP model reduced disease severity. (A-E) Representative clinical pictures on d 12 after injection of mice with rabbit anti-mCOL7C IgG and treatment with $(A, B)$ isotype control (shared with Figure 2) and (C,D) ETA every other day until d 10 starting 2 d prior to the initial anti-mCOL7C IgG injection. Both groups developed erythema and erosions.

(E) Clinical disease score, shown as the percentage of body surface area affected by blistering, was reduced in ETA-treated mice $(n=6)$ compared to isotype-treated mice with EBA $(n=8)$ at $d 8$ and d 12. (F) Representative pictures of mouse ears on $d 2$ after injection with rabbit anti-mCOL 17 IgG and treatment with PBS or ETA every other day starting 2 d prior to anti-mCOL17 lgG injection. (E) Clinical disease score, shown as the percentage of ear surface area affected by blistering, was reduced in ETA-treated mice $(n=6)$ compared to isotype-treated mice with EBA $(n=8)$ at $d 2\left(^{*} p<0.05\right.$, $\left.{ }^{* *} p<0.01\right)$.
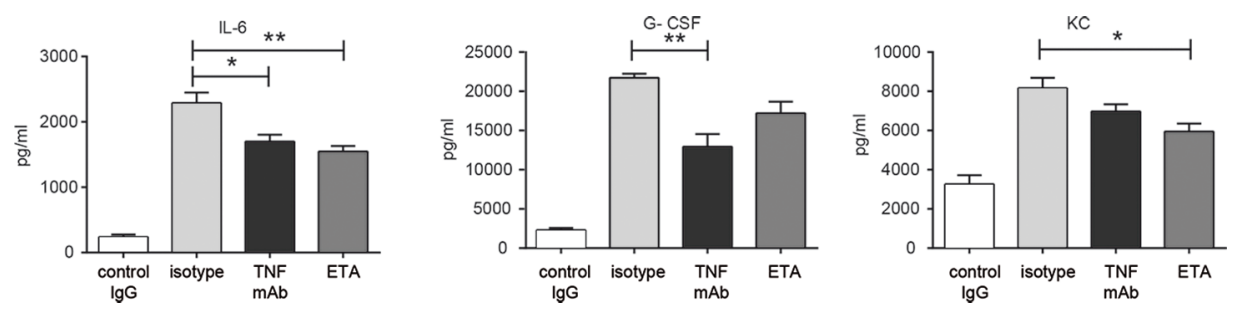

Figure 4. TNF inhibition in antibody transfer-induced EBA was associated with changes in serum levels of IL-6, G-CSF and KC. Circulating IL-6, G-CSF and KC levels were evaluated in murine serum at d 12 of antibody transfer-induced EBA using Bio-Plex. Statistically significant reduction of all three molecules was observed in the serum of mice with blocked TNF function (TNF mAb/ETA, $n=6$ ) compared with isotype-treated animals $\left(n=8 ;{ }^{*} p<0.05\right.$, $\left.{ }^{* *} p<0.01\right)$. disease activity in mice as a therapeutic approach. Taken together, these observations suggest that TNF is significantly involved in the effector phase of experimental EBA. Previously, different experimental studies demonstrated that TNF treatment was also effective in other AIBDs. More specifically, TNF plays a role in the acantholytic process in PV $(32,41)$, and its expression in serum and skin of patients with PV $(32,41)$ and BP (42) was increased. Furthermore, case reports documented the successful use of infliximab and ETA in PV patients (28-31), and in mucous membrane pemphigoid, the use of ETA was suggested for patients not responsive to high-dose intravenous immunoglobulins $(26,43)$. Interestingly, here we observed increased levels of TNF only at the site of inflammation (i.e., the skin), while TNF concentrations in the serum were identical in mice with or without experimental EBA. The reason for unchanged serum TNF expression in experimental EBA could be either too low concentration in the skin to affect serum expression or retention in the skin by binding to TNF receptors.

For TNF blockade, we employed MP6-XT22, a rat TNF-blocking IgG1 $\mathrm{mAb}$ that neutralizes murine TNF and is the standard TNF blocking antibody used in murine studies (44). The human reagent ETA is a TNFR2-FC fusion protein (soluble p75-TNF receptor-human IgG1) that is also effective at neutralizing TNF in mice and humans (44). Both compounds exhibited the same inhibitory effect on disease progression when applied in our model of antibody transfer-induced EBA. TNF signals via two receptors, TNFR1 and TNFR2. In contrast to TNFR1, which is ubiquitously expressed and mediates proinflammatory processes and cell death, TNFR2 is found on a limited number of cell types, such as cells of the hematopoietic lineage, endothelial cells and certain cells of the central nervous system (45), where it promotes tissue repair, angiogenesis and cell survival. TNFR2 is also involved in several immune diseases and inflammatory processes (10), 

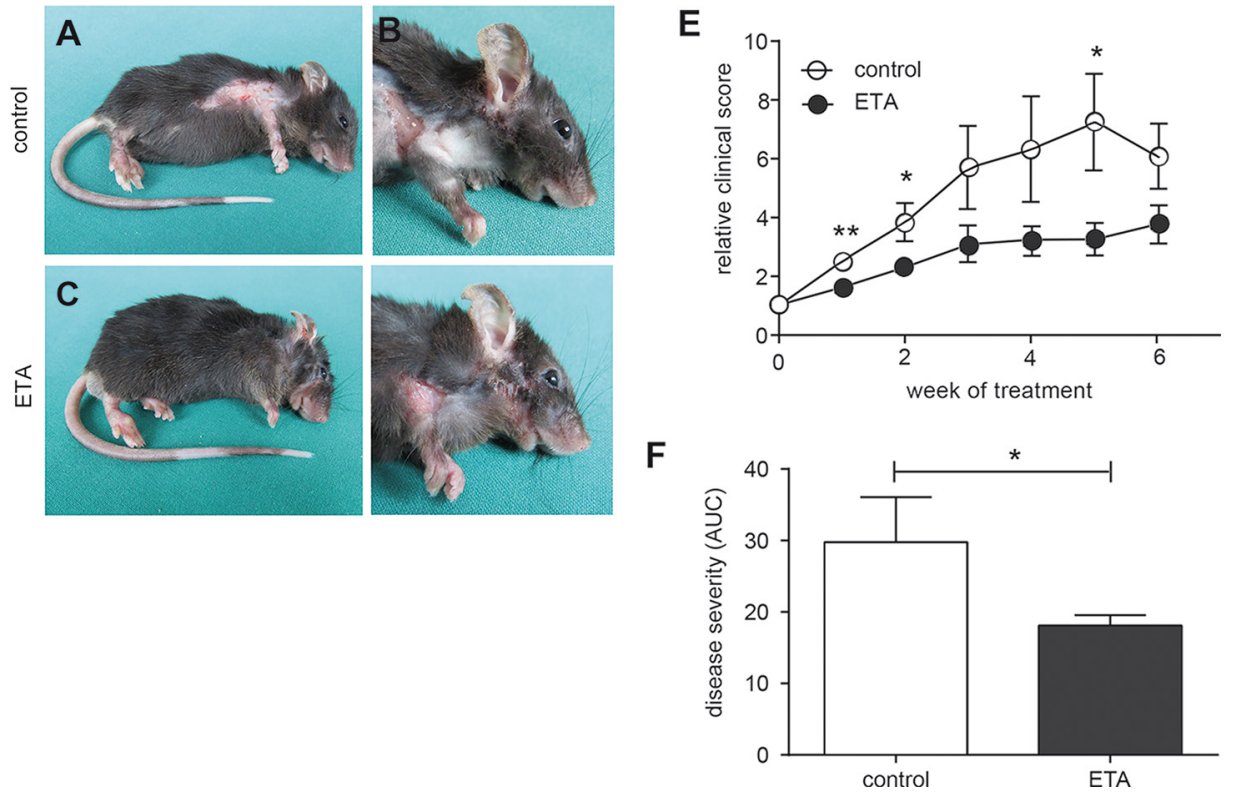

Figure 5. Therapeutic application of ETA improved disease severity in immunizationinduced EBA. Three to six weeks after B6.S mice were immunized with mCOL7C-GST in TiterMax, mice developed clinical disease with more than $2 \%$ affected skin and were allocated into either the PBS $(n=7)$ or ETA $(n=8)$ treatment group. Representative clinical pictures of mice at 6 wks of treatment that received $(A, B)$ PBS or $(C, D)$ ETA every second day for 6 wks. (E) Time course of clinical disease development, shown as percentage of body surface area affected by blistering and (F) as area under the curve calculated over time for the entire treatment period of 6 wks $\left({ }^{*} p<0.05,{ }^{* *} p<0.01\right)$.

which makes it likely to function as the major signaling receptor in EBA, thus is interesting for therapeutic modulation. However, which receptor primarily contributes to EBA development and progression is a subject of future research.

Our data indicate a reduced inflammatory milieu after TNF blockade that may contribute to improvement of skin disease. After use of TNF-blocking antibodies in antibody transfer-induced EBA, we observed strongly reduced TNF expression and reduced numbers of macrophages (MOMA-2-positive cells) in lesional skin. To date, the contribution of macrophages to the development of skin lesions in EBA, in both patients and experimental models, has been unclear. Here, we show that macrophages constitute a substantial percentage of infiltrated leukocytes in lesional skin obtained from mice with experimental EBA. In addition, serum cytokine levels of macrophage-released cytokines such as IL-6, G-CSF and the murine IL-8 equivalent keratinocytederived chemokine $(46,47)$ were reduced in mice with antibody transfer-induced EBA after therapy with ETA and/or TNF blockade, indicating a diseaserelated effect of macrophages in experimental EBA. To address the question of whether this increased number of macrophages in the skin of human and experimental AIBDs represents a bystander effect of tissue inflammation or actively contributes to disease expression, we analyzed the role of monocytes/macrophages during ROS release and split formation using human isolated monocytes. Indeed, here we show that isolated monocytes directly contribute to mechanisms important in the pathogenesis of EBA and other pemphigoid diseases.

In accordance with our data, it was previously shown that TNF blockade reduces cell infiltration in different autoimmune diseases, such as rheumatoid arthritis (48). In addition, it had been demonstrated that ETA, in contrast to other TNF inhibitors (such as infliximab), exerts its effect not by inducing apoptosis of macrophages (49) but by phosphorylating p38 MAPK and STAT3 and reducing complement-dependent lysis of macrophages (50). The reduced number of macrophages observed after use of TNF-blocking antibodies in antibody transfer-induced EBA may therefore be due to reduced infiltration of these cells.

\section{CONCLUSION}

In conclusion, increased TNF expression in the skin of mice with experimental EBA suggests that this cytokine may be a new potential therapeutic target. Furthermore, both ETA and TNF-blocking antibody significantly impaired skin blistering in a prophylactic setting of the antibody transfer-induced model of EBA. Moreover, therapeutic intervention of TNF activity was able to reduce disease activity in mice with fully developed skin lesions. Our results suggest blockade of TNF function as a new therapeutic approach for the treatment of EBA. In line with the fact that antibodies like sCD32, anti-Flightless-1 homolog, anti-GM-CSF and anti-FcgRIV have promising inhibitory effects in model systems of pemphigoid diseases, we believe that the therapy options for these difficult-to-treat autoimmune disorders will improve in coming years $(20,24,51,52)$.

\section{ACKNOWLEDGMENTS}

This study was supported by the following grants: Excellence Cluster "Inflammation at Interfaces" (EXC306/2); Research Training Group "Modulation of Autoimmunity" (GRK 1727/1); Clinical Research Unit "Pemphigoid Diseases" (KFO 303/1, project LU 877/11-1) and an unrestricted research grant from Abbott/Abbvie Deutschland GmbH and Co. KG. We thank Lidija Gutjahr and Marie-Luise Leppin for excellent technical assistance. 


\section{DISCLOSURE}

The authors declare that they have no competing interests as defined by Molecular Medicine, or other interests that might be perceived to influence the results and discussion reported in this paper.

\section{REFERENCES}

1. Carswell EA, Old LJ, Kassel RL, Green S, Fiore N, Williamson B. (1975) An endotoxininduced serum factor that causes necrosis of tumors. Proc. Nat. Acad. Sci. USA. 72:3666-70.

2. Kwiatkowski D, et al. (1990) TNF concentration in fatal cerebral, non-fatal cerebral, and uncomplicated Plasmodium falciparum malaria. Lancet. 336:1201-04.

3. Waage A, Halstensen A, Espevik T. (1987) Association between tumour necrosis factor in serum and fatal outcome in patients with meningococcal disease. Lancet. 1:355-57.

4. Armuzzi A, et al. (2014) Anti-TNF agents as therapeutic choice in immune-mediated inflammatory diseases: focus on adalimumab. Int. J. Immunopathol. Pharmacol. 27(1 Suppl):11-32.

5. Ben-Horin S, Kopylov U, Chowers Y. (2014) Optimizing anti-TNF treatments in inflammatory bowel disease. Autoimmunity Rev. 13:24-30.

6. Monaco C, Nanchahal J, Taylor P, Feldmann M. (2015) Anti-TNF therapy: past, present and future. Int. Immunol. 27:55-62.

7. Stidham RW, et al. (2014) Systematic review with network meta-analysis: the efficacy of anti-TNF agents for the treatment of Crohn's disease. Aliment Pharmacol. Ther. 39:1349-62.

8. Wendling D, Prati C. (2014) Paradoxical effects of anti-TNF-alpha agents in inflammatory diseases. Exp. Rev. Clin. Immunol. 10:159-69.

9. Cowin AJ, Hatzirodos N, Rigden J, Fitridge R, Belford DA. (2006) Etanercept decreases tumor necrosis factor-alpha activity in chronic wound fluid. Wound Repair Regen. 14:421-26.

10. Bradley JR. (2008) TNF-mediated inflammatory disease. J. Pathol. 214:149-60.

11. Finsterbusch M, Voisin MB, Beyrau M, Williams TJ, Nourshargh S. (2014) Neutrophils recruited by chemoattractants in vivo induce microvascular plasma protein leakage through secretion of TNF. J. Exp. Med. 211:1307-14.

12. Smart SJ, Casale TB. (1994) TNF-alpha-induced transendothelial neutrophil migration is IL-8 dependent. Am. J. Physiol. 266(3 Pt 1):L238-45.

13. Neta R, Sayers TJ, Oppenheim JJ. (1992) Relationship of TNF to interleukins. Immunol. Ser. 56:499-566.

14. Kasperkiewicz M, et al. (2016) Epidermolysis Bullosa Acquisita: From Pathophysiology to Novel Therapeutic Options. J. Invest. Dermatol. 136:24-33.

15. Witte M, Koga H, Hashimoto T, Ludwig RJ, Bieber K. (2016) Discovering potential drug targets for personalized treatment of autoimmune disorders: what we learn from epidermolysis bullosa acquisita. Exp. Opin. Ther. Targets. 20:985-98.

16. Ludwig RJ. (2013) Clinical presentation, pathogenesis, diagnosis, and treatment of epidermolysis bullosa acquisita. ISRN Dermatol. 2013:812029.

17. Ludwig RJ, Zillikens D. (2011) Pathogenesis of epidermolysis bullosa acquisita. Dermatol. Clin. 29:493-501, xi.

18. Gupta R, Woodley DT, Chen M. (2012) Epidermolysis bullosa acquisita. Clin. Dermatol. 30:60-69.

19. Ludwig RJ, Kalies K, Kohl J, Zillikens D, Schmidt E. (2013) Emerging treatments for pemphigoid diseases. Trends Mol. Med. 19:501-12.

20. Samavedam UK, et al. (2014) GM-CSF modulates autoantibody production and skin blistering in experimental epidermolysis bullosa acquisita. J. Immunol. 192:559-71.

21. Samavedam UK, et al. (20130) Recombinant IL-6 treatment protects mice from organ specific autoimmune disease by IL-6 classical signalling-dependent IL-1ra induction. J. Autoimmun. 40:74-85.

22. Mihai S, et al. (2007) The alternative pathway of complement activation is critical for blister induction in experimental epidermolysis bullosa acquisita. J. Immunol. 178:6514-21.

23. Chiriac MT, et al. (2007) NADPH oxidase is required for neutrophil-dependent autoantibody-induced tissue damage. J. Pathol. 212:56-65.

24. Kasperkiewicz M, et al. (2012) Genetic identification and functional validation of FcgammaRIV as key molecule in autoantibody-induced tissue injury. J. Pathol. 228:8-19.

25. Grando SA, et al. (1989) Mediators of inflammation in blister fluids from patients with pemphigus vulgaris and bullous pemphigoid. Arch. Dermatol. 125:925-30.

26. John H, Whallett A, Quinlan M. (2007) Successful biologic treatment of ocular mucous membrane pemphigoid with anti-TNF-alpha. Eye. 21:1434-35.

27. Kennedy JS, Devillez RL, Henning JS. (2010) Recalcitrant cicatricial pemphigoid treated with the anti-TNF-alpha agent etanercept. J. Drugs Dermatol. 9:68-70.

28. Berookhim B, Fischer HD, Weinberg JM. (2004) Treatment of recalcitrant pemphigus vulgaris with the tumor necrosis factor alpha antagonist etanercept. Cutis. 74:245-47.

29. Jacobi A, Shuler G, Hertl M. (2005) Rapid control of therapy-refractory pemphigus vulgaris by treatment with the tumour necrosis factor-alpha inhibitor infliximab. Brit. J. Dermatol. 153:448-49.

30. Pardo J, et al. (2005) Infliximab in the management of severe pemphigus vulgaris. Brit. J. Dermatol. 153:222-23.

31. Prajapati V, Mydlarski PR. (2008) Advances in pemphigus therapy. Skin Ther. Lett. 13:4-7.

32. Feliciani C, et al. (2000) In vitro and in vivo expression of interleukin-1alpha and tumor necrosis factor-alpha mRNA in pemphigus vulgaris: interleukin-1alpha and tumor necrosis factor-alpha are involved in acantholysis. J. Invest. Dermatol. 114:71-77.
33. Sitaru C, et al. (2005) Induction of dermalepidermal separation in mice by passive transfer of antibodies specific to type VII collagen. J. Clin. Invest. 115:870-78.

34. Iwata H, et al. (2013) B cells, dendritic cells, and macrophages are required to induce an autoreactive CD4 helper T cell response in experimental epidermolysis bullosa acquisita. J. Immunol. 191:2978-88.

35. Sitaru C, et al. (2006) Induction of complement-fixing autoantibodies against type VII collagen results in subepidermal blistering in mice. J. Immunol. 177:3461-68.

36. Schulze FS, et al. (2014) Fcgamma receptors III and IV mediate tissue destruction in a novel adult mouse model of bullous pemphigoid. Am. J. Pathol. 184:2185-96.

37. Hammers CM, et al. (2011) Complement-fixing anti-type VII collagen antibodies are induced in Th1-polarized lymph nodes of epidermolysis bullosa acquisita-susceptible mice. J. Immunol. 187:5043-50

38. Recke A, et al. (2014) Recombinant human IgA1 and IgA2 autoantibodies to type VII collagen induce subepidermal blistering ex vivo. J. Immunol. 193:1600-08.

39. Kasprick A, et al. (2015) Conditional depletion of mast cells has no impact on the severity of experimental epidermolysis bullosa acquisita. Eur. J. Immunol. 45:1462-70.

40. Hellberg L, et al. (2013) Methylprednisolone blocks autoantibody-induced tissue damage in experimental models of bullous pemphigoid and epidermolysis bullosa acquisita through inhibition of neutrophil activation. J. Invest. Dermatol. 133:2390-99.

41. Lopez-Robles E, et al. (2001) TNFalpha and IL-6 are mediators in the blistering process of pemphigus. Int. J. Dermatol. 40:185-88.

42. D'Auria L, et al. (1999) Increased serum IL-6, TNF-alpha and IL-10 levels in patients with bullous pemphigoid: relationships with disease activity. J. Eur. Acad. Dermatol. Venereol. 12:11-15.

43. Sobolewska B, Deuter C, Zierhut M. (2013) Current medical treatment of ocular mucous membrane pemphigoid. Ocul. Surf. 11:259-66.

44. Plessner HL, et al. (2007) Neutralization of tumor necrosis factor (TNF) by antibody but not TNF receptor fusion molecule exacerbates chronic murine tuberculosis. J. Infect. Dis. 195:1643-50.

45. Faustman DL, Davis M. (2013) TNF Receptor 2 and Disease: Autoimmunity and Regenerative Medicine. Front. Immunol. 4:478.

46. Aoki Y, Hirano D, Kodama H, Nishi Y, Nakamura M. (1998) Stimulation of G-CSF gene expression in the macrophage cell line by contact with extracellular matrix proteins and a pre-B leukaemia cell line. Cytokine. 10:596-602.

47. Arango Duque G, Descoteaux A. (2014) Macrophage cytokines: involvement in immunity and infectious diseases. Front. Immunol. 5:491.

48. Smeets TJ, Kraan MC, van Loon ME, Tak PP. (2003) Tumor necrosis factor alpha blockade 
INF INHIBITION IN EXPERIMENTAL EBA

reduces the synovial cell infiltrate early after initiation of treatment, but apparently not by induction of apoptosis in synovial tissue. Arthritis Rheum. 48:2155-62.

49. Van den Brande JM, et al. (2003) Infliximab but not etanercept induces apoptosis in lamina propria T-lymphocytes from patients with Crohn's disease. Gastroenterology. 124:1774-85.

50. Wong M, et al. (2008) TNFalpha blockade in human diseases: mechanisms and future directions. Clin. Immunol. 126:121-36.

51. Iwata $\mathrm{H}$, et al. (2015) Recombinant soluble CD32 suppresses disease progression in experimental epidermolysis bullosa acquisita. J. Invest. Dermatol. 135:916-19.

52. Kopecki Z, et al. (2013) Topically applied flightless I neutralizing antibodies improve healing of blistered skin in a murine model of epidermolysis bullosa acquisita. J. Invest. Dermatol. 133:1008-16.

Cite this article as: Hirose M, et al. (2016) Reduced skin blistering in experimental epidermolysis bullosa acquisita after anti-TNF treatment. Mol. Med. 22:918-26. 